\title{
Argumentando sobre a realidade dos potenciais
}

\author{
(Arguing on the actuality of the potentials)
}

\author{
G.F. Leal Ferreira \\ Universidade de São Paulo, Instituto de Física de São Carlos, São Carlos, SP, Brasil. \\ Recebido em 8/5/03; Aceito em 7/1/04
}

\begin{abstract}
Argumenta-se que, da mesma forma que o efeito Aharonov-Bohm mostrou ser o potencial vetor detetável microscopicamente, os nossos transformadores parecem indicar que ele o é macroscopicamente através de sua derivada temporal. Nessa suposição, são estudadas as transformações de calibre a partir do calibre de Lorentz, que é o calibre causal. Os potenciais, escalar e vetorial, de uma carga em movimento nos calibres de Coulomb e Lorentz são apresentados. A ação mecânica dos potenciais também é discutida, mostrando-se que os efeitos de indução entre circuitos são incluidos no momento canônico na formulação de Hamilton. Finalmente menciona-se que a mais rica Álgebra Geométrica (ou Geometria de Clifford) é mais adequada para a descrição do Eletromagnetismo.
\end{abstract}

Palavras-chave: potencial escalar, potencial vetor, transformações de calibre, efeito Aharonov-Bohm.

It is argued that inasmuch as the Aharonov-Bohm effect supports the view on the actuality of the vector potential at the microscopic level, by the same token our very familiar transformers seems to perform the same at the macroscopic level through its time dependence. Assuming this, gauge transformations are studied proceeding from the causal Lorentz gauge. The potentials of a moving charge in the Coulomb and Lorentz gauges are presented. The mechanical action of the potentials is also discussed, and it is shown that induction effects between line currents are included in the canonical momentum of the Hamiltonian formalism. It is finally argued that the reacher Geometric (Clifford) Algebra is more suited for description of the Electromagnetism.

Keywords: scalar potential, vector potential, Gauge transformations, Aharonov-Bohm effect.

\section{Introdução}

No desenvolvimento do Eletromagnetismo dois conceitos emergiram, o de campo elétrico e magnético (omitindo 'de indução') e o de seus potenciais, escalar e vetorial. Maxwell, fazendo-se intérprete das idéias de Faraday, considera o campo elétrico entre os condutores carregados, como sítio dos fenômenos elétricos, inclusive tensionando o éter [1]. Apesar disso, ao estudar as correntes de indução eletromagnética, atribui ao negativo da variação temporal do potencial vetor (este chamado por ele de 'momento cinético' [1]) a força eletromotriz da corrente de indução. Para ele, 'electromotive force is always to be understood to act on electricity only, not on the bodies in which electricity resides' [1] (força eletromotriz deve ser sempre entendida como agindo sobre cargas elétricas e não sobre os corpos em que elas residem). Com a sistematização da teoria, os conceitos de campo elétrico e magnético ganharam foro de grandezas físicas [2], ficando os potenciais como utilíssimas ferramentas matemáticas, mas não mais que isso. Em boa parte isto proveio do fato de serem os potenciais um tanto arbitrários, já que diferentes duplas de potencial vetor e potencial escalar, darem origem a um único par de campo elétrico e magnético. As transformações de calibre (gauge) geram aquela variedade. Porém, descobriu-se posteriormante que no mundo microscópico o potencial vetor mos-

Enviar correspondência para G.F. Leal Ferreira. Universidade de São Paulo, Instituto de Física de São Carlos, DFCM, C.P. 369, 13.560-970 São Carlos, SP, Brasil. E-mail: guilherm@if.sc.usp.br. tra ter sentido físico, no efeito Aharonov-Bohm [3-5], modificando a interferência entre feixes eletrônicos coerentes que atravessam as duplas fendas da experiência clássica. O efeito, porém, deixa indecidida a questão do calibre, declarando aqueles autores [3] "insofar as the potentials are richer in properties than fields, there is no way to reveal this additional richness" (apesar de os potenciais serem mais ricos em propriedades que os campos, não há como revelar esta riqueza adicional ). Isto é, a experiência não nos diria qual calibre é o calibre físico. Do nosso ponto de vista, caberia a nós, na medida do possível, dizer qual é o calibre físico.

A experiência de Aharonov-Bohm valhe-se do fato de o potencial vetor de um imã cilíndrico ser não nulo no seu exterior onde o campo magnético é zero (Figura 1), com o raciocínio de que uma grandeza nula numa região do espaço, o campo magnético, não pode agir aí físicamente. A ação do ímã deveria pois ser imputada ao potencial vetor. A diferença de sentido do potencial vetor nas duas trajetórias que passam pelas fendas causa diferença de fase nas funções de onda que se interferem no anteparo. Mas, como discutiremos na próxima seção, o que ocorre nos conhecidos transformadores, Figura 2, não é diferente. Embora se trate aquí de fenômeno dependente do tempo, o potencial vetor guarda a mesma relação com as correntes como na experiência de Aharonov-Bohm e como será discutido na próxima seção, não seria mais natural atribuir a indução ao potencial vetor? Admitindo isto, vamos nos lembrar que entre os calibres há o calibre causal dos potenciais retarda- 


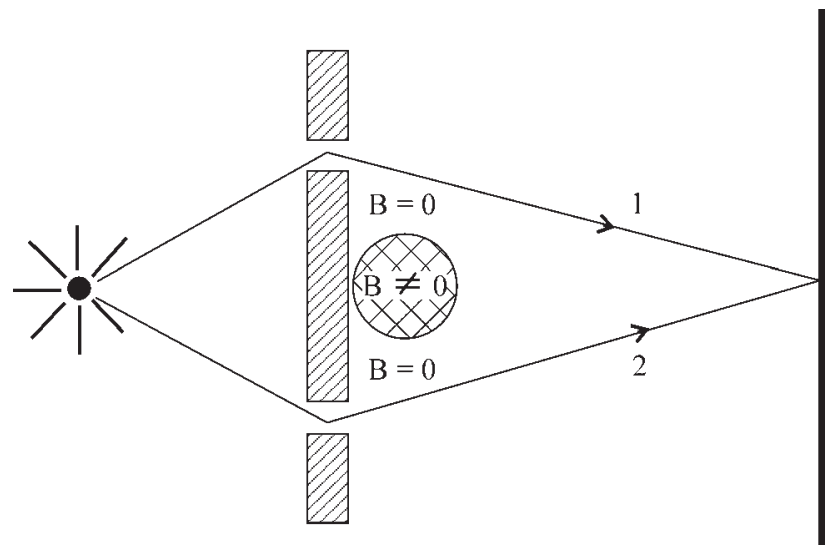

Figura 1 - Esquema idealizado da experiência proposta por AharonovBohm. Entre as fendas, está colocado ímã, cujo campo magnético $B$, como se sabe, só é diferente de zero no seu interior, $B \neq 0$. Já o potencial vetor, diferente de zero em todo o espaço, com simetria cilíndrica, causa efeitos opostos nas tajetórias 1 e 2 (adaptada de [4]).

dos, chamado de calibre de Lorentz, no qual os potenciais se propagam a partir de suas fontes, cargas e correntes. Como entre estas há o vínculo da equação da continuidade, entre os potenciais aparece o vínculo da condição de Lorentz, ligando a divergência do vetor potencial à variação no tempo do potencial escalar. Daremos ao calibre de Lorentz sentido físico primordial, e estudaremos as transformações de calibre que partem do par causal.

Tentativamente diríamos que os potenciais 'existem' no vácuo, e que os campos, elétrico e magnético, 'materializam-se' em presença de cargas. Ou seja, os 'campos' gerados pelas cargas são os potenciais; a presença de uma carga extra 'materializa' os campos elétrico e magnético. Não estaremos aquí empenhados em 'provar' esta visão, mesmo porque não o podemos fazer, mas no pressuposto convincente da realidade dos potenciais, procuraremos nos familiarizar com o par, vetor e escalar, apresentando-os em algumas situações de interesse.

\section{O Argumento sobre a realidade macroscópica do potencial vetor}

Qual é a explicação 'ortodoxa' sobre o funcionamento do transformador? É a seguinte: a variação temporal do fluxo magnético no núcleo do transformador, Figura 2, cria no seu exterior o campo elétrico de indução (no sentido negativo) da mesma forma que uma corrente fechada que circulasse ao longo do núcleo criaria um campo magnético (agora no sentido positivo) no exterior. O campo de indução cria então a corrente que circula na espira C. Em relação à experiência de Aharonov-Bohm, a ortodoxia aceita com alguma perplexidade que o potencial vetor tem aí uma ação.

Voltemos à Figura 1 e imaginemos que em vez do ímã tivéssemos ali um solenóide, alimentado por corrente dependente do tempo, como fonte do campo. Se a variação da corrente fosse suficientemente lenta, obteríamos padrões de interferência correspondentemente variáveis, perturbados pela presença do campo elétrico de indução sobre os elétrons passando pelas fendas. Seja outra vez a Figura 2 e

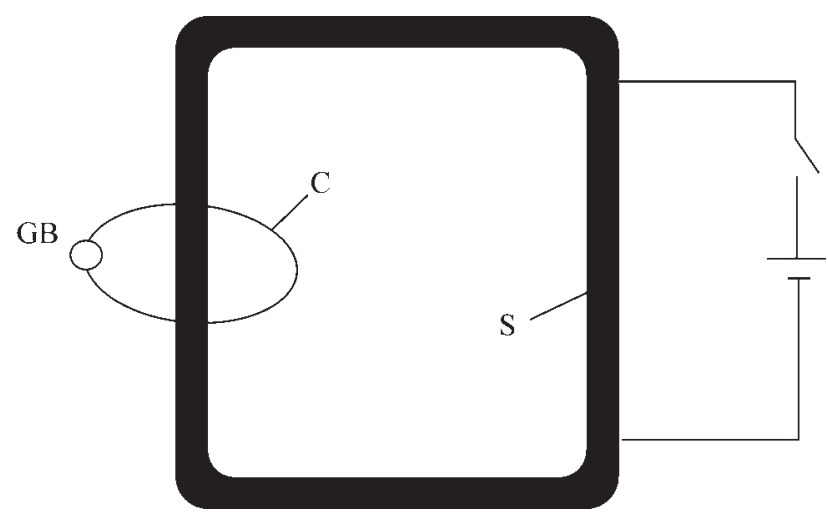

Figura 2 - O solenóide $\mathrm{S}$, do primário, ao ser excitado por corrente, gera na espira de raio $r$, munida de um galvanômetro balístico GB, corrente na espira C. O campo magnético $\vec{B}$ é nuzlo no exterior do circuito de ferro, hachureado, enquanto que o potencial vetor não o é, e a ele deve ser atribuida a indução da corrente $i$. $R$ é a resistência total da espira e do galvanômetro balístico (adaptada de [5]).

comparemô-la à Figura 1. Vemos que a diferença essencial entre elas é que na região exterior ao 'solenóide' (região de campo magnético), enquanto que na Figura 1 temos fendas por onde passam elétrons, na Figura 2 temos o circuito $\mathrm{C}$, sensível à variação do potencial vetor. Quer dizer, uma, sensível ao próprio potencial vetor, e a outra, à sua derivada temporal. Como argumentado em [5], não é a visão da atualidade do potencial vetor defendida aqui bem mais consistente e econômica do que a 'ortodoxa' em que ambos os conceitos são primordiais?

\section{Transformações de calibre}

Entre as objeções à realidade do potenciais está a de sua arbitrariedade em face às transformações de calibre. Devemos, pois, discutir o assunto.

Em termo dos potenciais, os campos $\vec{E}$ e $\vec{B}$ são obtidos do par de potenciais $\vec{A}$ e $U$ como

$$
\vec{B}=\nabla \times \vec{A}
$$

e, $\operatorname{com} c$ a velocidade da luz,

$$
\vec{E}=-\nabla \vec{U}-\frac{1}{c} \frac{\partial U}{\partial t}
$$

esta última relação decorrendo do fato de que

$$
\nabla \times \vec{E}=-\frac{1}{c} \frac{\partial \vec{B}}{\partial t}
$$

Agora, do par $\vec{A}, U$ podemos obter outro par, $\vec{A}, U$ ', produzindo os mesmos campos que $\vec{A}, U$, através da transformação de calibre com a função geradora $(f . g.) \Lambda$

$$
\vec{A}{ }^{\prime}=\vec{A}+\nabla \Lambda
$$

$\mathrm{e}$

$$
U^{\prime}=U-\frac{1}{c} \frac{\partial \Lambda}{\partial t}
$$

o que pode facilmente ser constatado pelo uso das Eqs. $1 \mathrm{e}$ 2. $\Lambda$ é uma função arbitrária de $\vec{x}$ e $t$, sem nenhum vínculo 
necessário com as fontes do problema em questão. A Eq. 1 deixa antever uma certa liberdade de escolha de $\vec{A}$, já que só seu rotacional está determinado.

\subsection{Transformações de calibre causais}

Como é bem conhecido [4], fixando em especial a divergência do potencial vetor $\vec{A}_{L}$ através de

$$
\nabla \cdot \vec{A}_{L}+\frac{1}{c} \frac{\partial U_{L}}{\partial t}=0
$$

em que $U_{L}$ é o correspondente potencial escalar, está-se no calibre causal de Lorentz, em que os potenciais $\vec{A}_{L}$ e $U_{L}$ propagam-se a partir de suas fontes, que são as densidades de carga e de corrente. Por causa desse importante caráter físico, tomaremos, em geral, o par $\vec{A}_{L}$ e $U_{L}$, satisfazendo a Eq. 6, como par de partida para construção de novos pares $\vec{A}_{L}, U$. Então, vamos por

$$
\vec{A}=\vec{A}_{L}+\nabla \Lambda
$$

e

$$
U=U_{L}-\frac{1}{c} \frac{\partial \Lambda}{\partial t}
$$

Como estamos adotando ponto de vista realista para os potenciais, seria interessante que a $f . g . \Lambda$ nas Eqs. 7 e 8 mantivesse um mínimo de correlação, mesmo que só matemática, com os potenciais $\vec{A}_{L}$ e $U_{L}$ para garantir que $\vec{A}$ e $U$ também o tenham. Num primeiro caso, achemos a divergência dos dois lados da Eq. 7, $\operatorname{com} \vec{A} \rightarrow \vec{A}_{C}$ e $U \rightarrow U_{C}$. Chamando a f.g. de $\Lambda_{L C}$ e usando a Eq. 6,

$$
\nabla \cdot \vec{A}_{C}=-\frac{1}{c} \frac{\partial U_{L}}{\partial t}+\nabla^{2} \Lambda_{L C}
$$

A única maneira de fazermos uma escolha que force $\Lambda_{L C}$ se relacionar só com as fontes é impor $\nabla \cdot \vec{A}_{C}=0$ ou seja,

$$
\nabla^{2} \Lambda_{L C}=\frac{1}{c} \frac{\partial U_{L}}{\partial t}
$$

Aplicando-se o laplaciano aos dois membros da Eq. 8, e usando a Eq. 10, vem

$$
\nabla^{2} U_{C}=\nabla^{2} U_{L}-\frac{1}{c^{2}} \frac{\partial^{2} U_{L}}{\partial t^{2}}(=-4 \pi \rho)
$$

completando o lado direito da Eq. 11 com relação conhecida [2]. $\rho$ é a densidade de carga, mostrando que $U_{C}$, o novo potencial, é instantâneo. Esse é o calibre de Coulomb, daí o índice $\mathrm{C}$.

Uma outra maneira de se obter $f . g ., \Lambda_{\mathrm{LP}}$, relacionada às fontes é na Eq. 8 impormos $U_{P}=0$. Então,

$$
\frac{\partial \Lambda_{L P}}{\partial t}=c U_{L}
$$

e, integrando no tempo para $x$ fixo,

$$
\Lambda_{L P}=c \int_{o}^{t} U_{L} d t
$$

e a Eq. 7 dá

$$
\overrightarrow{\mathrm{A}}_{P}=\overrightarrow{\mathrm{A}}_{L}+c \int_{o}^{t} \nabla U_{L} d t
$$

Portanto, nesse calibre, prescindimos do potencial escalar. Os campos elétrico e magnético derivam somente do potencial vetor. Em [6], tratando da criação instantânea de um dipolo, o correspondente potencial vetor foi chamado de proto-potencial vetor. Como notado em [7], a descrição por meio do potencial eletrostático (isto é, $U_{L} \neq 0$ e $\vec{A}_{L}=0$ ) de uma distribuição estática de carga pode ser substituida pela do potencial vetor, que aparentemente se reporta ao processo de criação (ver Eq. 13 de [6]).

Um caso particular interessante de ser discutido separadamente é aquele em que temos $U_{L}=0$ e $\vec{A}_{L} \neq 0$, ou seja, trata-se de campo criado por correntes fechadas, estacionárias ou não, mas satisfazendo $\nabla \cdot \vec{A}_{L}=0$. Pela Eq. 10, sendo $U_{L}=0, \nabla^{2} \Lambda_{L C}=0$ e não haveria maneira de escolher $\Lambda_{L C} \neq 0$ relacionado aos potenciais causais. Então, tem-se $\nabla$. $\vec{A}_{L}=\nabla \cdot \vec{A}_{C}=0$ ou, essencialmente, $\vec{A}_{L}=\vec{A}_{C}$.

\subsection{Uma relação útil}

Dispondo dos pares $\vec{A}_{C}, U_{C}$ e $\vec{A}_{P}, U_{P}(=0)$, podemos fazer a transformação direta $P \rightarrow C$ através de $\Lambda_{P C}$

$$
\overrightarrow{\mathrm{A}}_{C}=\overrightarrow{\mathrm{A}}_{P}+\nabla \Lambda_{P C}
$$

e como $U_{P}=0$, segue da Eq. 5 que

$$
U_{C}=-\frac{1}{c} \frac{\partial \Lambda_{P C}}{\partial t}
$$

$\mathrm{ou}$

$$
\Lambda_{P C}=-c \int_{0}^{t} U_{C} d t
$$

Usando-se agora a Eq. 14, chegamos a uma relação entre os calibres de Lorentz e Coulomb envolvendo somente integrais no tempo

$$
\vec{A}_{C}=\vec{A}_{L}+c \nabla \int_{0}^{t}\left(U_{L}-U_{C}\right) d t
$$

\section{Os potenciais de uma carga em movimento}

As equações de Maxwell são escritas para os campos elétrico e magnético. Nesta forma, elas escondem o papel importante que joga o retardo na teoria eletromagnética, o qual só vem a se explicitar nas equações dos potenciais. É o retardo, por exemplo, que torna o rotacional do campo elétrico de uma carga em movimento uniforme não nulo. Vale a pena aquí chamar a atenção outra vez [8] da guinada que o ensino do Eletromagnetismo dá ao estudar a indução eletromagnética. Enquanto que na Eletrostática e na Magnetostática os campos de um sistema de cargas, paradas ou 
em movimento, são vistos como uma superposição dos campos de cada uma das cargas, pelo menos implicitamente, para o estudo da indução, são introduzidas as equações dos campos, em especial, a equação da Lei de Faraday (a do rotacional do campo elétrico). Então, a indução de um circuito num outro vizinho é agora explicada por efeitos globais, ou seja, o da variação temporal do fluxo magnético ‘compreendido' pelo circuito vizinho. O ensino atual não se importa em restaurar a linha seguida anteriormente, baseada na superposição das ações individuais das cargas. Ritz e O 'Rahilly $[9,10]$ porém o fizeram, e mostraram que os efeitos de indução derivam da aceleração das cargas do circuito indutor (ver também [8]). Mas essa restauração, importante do ponto de vista conceitual, só foi possível porque ambos partiram de equações causais, os potenciais retardados de Liénard-Wiechert, desenvolvidos numa certa aproximação. Recentemente chegamos ao mesmo resultado, mas por caminho bem diferente [8]. Obtivemos o sistema consistente de equações de Maxwell que descrevem os estados quase-permanentes - que incluem os efeitos de indução -, e os potenciais dos quais os campos derivam. Essencialmente, a aproximação dos estados quase-permanentes consiste em usar o campo eletrostático na corrente de deslocamento (em vez do campo elétrico total), o que garante ser a solução correta até a ordem de $v^{2} / c^{2}$, sendo $v$ a velocidade das cargas. Deduzimos então o potencial vetor de uma carga em movimento e o campo elétrico resultante (que é o campo coulombiano corrigido pela velocidade e aceleração da carga), que coincidiu com aquele deduzido por Ritz e O 'Rahilly $[9,10]$ a partir dos potenciais de Liénard-Wiechert, desenvolvidos até a ordem de $v^{2} / c^{2}$ e até distâncias $r$ tais que $a r / c^{2}<<1$, sendo $a$ a aceleração da carga. O ponto interessante aquí é que o potencial vetor de onde partimos para depois obtermos os campos tem divergência nula, ou seja, está no calibre de Coulomb e não no de Lorentz. Ou seja, aquela maneira didática que comumente usamos de incorporarmos a lei da indução ao Eletromagnetismo, pela qual adicionamos ao campo eletrostático (instantâneo) um outro campo elétrico solenoidal, leva diretamente ao calibre de Coulomb.

O potencial escalar de uma carga $q$ em repouso é o familiar $q / r$ e o potencial vetor é nulo. Com a carga em movimento, com velocidade $\vec{v}$, entra em ação o potencial vetor, com contribuição da ordem de $q \vec{v} / c r$. A velocidade da luz, no denominador, dimensiona o valor de sua ação como potencial, a qual se assemelha a de um 'vento direcionado de potencial'. Mais exatamente, as expressões dos potenciais até a ordem de $v^{2} / c^{2}$ e para $a r / c^{2}<<1$, no calibre de Lorentz, $\vec{A}_{L}$ e $U_{L}$, são

$$
\vec{A}_{L} \simeq \frac{q \vec{v}}{c r}
$$

e

$$
U_{L} \simeq \frac{q}{r}\left(1+\frac{v^{2}}{2 c^{2}}-\frac{(\hat{r} \cdot \vec{v})^{2}}{2 c^{2}}\right)-\frac{q \vec{a}}{2 c^{2}}
$$

como calculado por Ritz e O'Rahilly $[9,10]$. No calibre de Coulomb, $U_{C}$ e $\vec{A}_{C}$, as coisas se invertem e o potencial escalar agora é instantâneo, enquanto o potencial vetor não o é,

$$
U_{C}=\frac{q}{r}
$$

e

$$
\vec{A}_{C} \simeq \frac{q}{2 c}\left(\frac{\vec{v}}{r}+\frac{\vec{r} \hat{r} \cdot \vec{v}}{r^{2}}\right)
$$

como calculado em [8]. Nas Eqs. 19-22, o retardo já foi eliminado e o tempo é o atual, com $\hat{r}$ é o versor da direção $\vec{r}$. Essas equações referem-se aos potenciais de uma carga em movimento, válida até a ordem de $v^{2} / c^{2}$ e para $\operatorname{ar} / c^{2}<<1$.

O potencial vetor exato no calibre de Coulomb $\vec{A}_{C}$ de uma carga em movimento pode ser obtido do potencial vetor de Liénard- Wiechert $\vec{A}_{L W}$ com o auxílio da Eq. 19 e da Eq. 33 de [8], em que se calcula a derivada do tempo atual em relação ao tempo retartado. Obtém-se para $\vec{A}_{C}$,

$$
\vec{A}_{C}=\vec{A}_{L W}+c q \nabla \int_{o}^{t}\left(\frac{1}{r_{r}}-\frac{1}{r}\right) d t
$$

em que $r_{r}$ é distância retardada e o tempo é o atual.

\section{A ação mecânica dos potenciais}

Embora os campos dêem diretamente as forças e entrem nas equações de movimento através da força de Lorentz, os potenciais fazem o mesmo de outra maneira, através de potenciais dependentes da velocidade. Como é bem sabido [11], a Lagrangeana $L$ de uma carga $q$ movendo-se no campo dos potenciais $\vec{A}, U$ é

$$
L=T-q U+\frac{q}{c} \vec{A} \cdot \vec{v}
$$

em que $T$ é a energia cinética e o calibre dos potenciais é qualquer. Passando à equação de movimento, tem-se

$$
\frac{d}{d t}\left(m \vec{v}+\frac{q}{c} \vec{A}\right)=-q \nabla\left(U-\frac{\vec{v} \cdot \vec{A}}{c}\right)+\vec{f}
$$

onde adicionamos $\vec{f}$, uma força de natureza não eletromagnética eventualmente presente. Notemos que $\nabla(\vec{v} \cdot \vec{A})=(\vec{v} . \nabla)$ $\vec{A}+\vec{v} x(\nabla x \vec{A})$. Visivelmente o termo $m \vec{v}+(q / c) \vec{A}$ faz agora o papel de momento da carga e no tratamento Hamiltoniano será o momento canônico, que por sua vez se transformará em $-i h \nabla / 2 \pi$ no tratamento do efeito Aharonov-Bohm. Konopinski [12] chama o termo $(q / c) \vec{A}$ de estoque de momento de campo à disposição da carga (store of field momentum available to the charge) porque na ausência de gradientes no lado direito da Eq. 25, o lado esquerdo é análogo ao da equação de variação da energia cinética

$$
\frac{d}{\partial t}(T+q U)=0
$$

em que $q U$ pode ser considerado como o estoque de energia de campo à disposição da carga. Adaptando o raciocínio à 
nossa Figura 2, Konopinski [12] argumenta que o espaço onde se situa a espira $C$ propiciaria a medida de $A$ : ao se fazer passar corrente no solenóide, uma carga, inicialmente em repouso e constrangida a se mover com raio constante, adquiriria velocidade, que seria uma medida do valor $A$. Aliás, com menos sofisticação, o mesmo poderia ser conseguido através do circuito da Figura 2: o galvanômetro balísitico GB ou um eletrômetro operando no modo carga mediria a carga circulada $Q$, que seria também uma medida de $A$ na espira $C$. De fato, em circuitos elétricos o termo de massa na Eq. 20 é pequeno em relação ao termo em A e sobre as cargas em movimento agem forças de resistência $\vec{f}$. Teríamos, com o termo em $\nabla(\vec{v} \cdot \vec{A})$ nulo na Eq. 20 ,

$$
\frac{1}{c} \frac{d}{d t} \oint A d l=R i
$$

em que $R$, devido a $\vec{f}$, é a resistência total, da espira com o galvanômetro balístico, e $i$ a corrente em C. Integrando ao longo da espira circular de raio $r$, temos

$$
2 \pi r A=c R \int_{0}^{\infty} i d t=c R Q
$$

Nesta equação, $A$ será o valor final alcançado pelo potencial vetor na espira devido ao circuito indutor.

Alargando nossa visão, chega-se à conclusão que os efeitos de indução em circuitos fixos, e mesmo os de autoindução, são descritos na linguagem Hamiltoniana pelas variações do momento cinético integrado ao longo do circuito. Por outro lado, os efeitos de indução devido a movimentos relativos entre circuitos são descritos pelo termo em $\nabla(\vec{v} . \vec{A})$, sendo $\vec{v}$ a velocidade de translação.

\section{Considerações finais}

Na década de 1960, era comum ouvir-se que não se devia fazer 'imagens' das grandezas elétricas, porque incorria-se no risco de mecanizá-las. Mas, por outro lado, não fazer nenhuma imagem é tratá-las todas como entidades matemáticas. Por exemplo, tentar separar os papéis que $\vec{B}$ e $\vec{H}$ jogam no magnetismo da matéria, ou seja entender porque a magnetização induzida está ligada ao $\vec{H}$ e não a $\vec{B}$ é para nós relevante e para isso, há que se ir além da visão matemática e construir imagens [13]. No caso presente, a identificação dos potenciais como entidades mais primitivas do que os campos tem certamente relevância, embora não se possa ainda saber se disso outras consequências advirão.

O par híbrido potencial escalar- potencial vetor é uma entidade perfeitamente bem definida no quadro da Álgebra Geométrica (ou de Clifford) [14,15], como um multivetor ou clifor, 0 -1, isto é, uma entidade constituida da 'soma' de um escalar e de um vetor (como um quatérnio [14]). Já o campo elétromagnético é do tipo 1-2, constituido da 'soma' de um vetor, o campo elétrico, e de um bi-vetor, gerador do campo magnético. Não há dúvida que a Álgebra Geométrica, com sua rica ontologia e estrutura, é muito mais apropriada à descrição dos fenômenos eletromagnéticos do que a Álgebra de Gibbs, que é aquela usualmente empregada, inclusive aquí. Talvez seja necessário algum esforço para conseguir tornar a Álgebra Geométrica um pouco mais física.

\section{Referências}

[1] J.C. Maxwell, A Treatise on Electricity and Magnetism (Dover, 1954), v. 1 e 2.

[2] R.P. Feynman, R.B. Leighton e M. Sands, The Feynman's Lectures (Addison-Wesley, 1966), v. 1 e 2.

[3] Y. Aharonov e D. Bohm, Phys. Rev. 115, 485 (1959).

[4] J.J. Sakurai, Advanced Quantum Mechanics (Addison-Wesley, 1967), cap. 1.

[5] R. Schlegel, Superposition \& Interaction (The Univ. of Chicago Press, 1980), cap. 6.

[6] G.F. Leal Ferreira, Rev. Bras. Ens. Física 20, 201 (1998).

[7] D.H. Kobe, Amer. J. Physics 49, 1075, (1981).

[8] G.F. Leal Ferreira, Rev. Bras. Ens. Física 23, 395 (2001).

[9] W. Ritz, Recherches Critiques sur l 'Életrodynamique Générale (Gauthier Villars, 1911), p. 382.

[10] A. O'Rahilly, Electromagnetic Theory (Dover, 1965), v. 1, cap. 7.

[11] H. Goldstein, Classical Mechanics (Addison-Wesley, 1951), cap. 1.

[12] E.J. Konopinski, Amer. J. Physics 46, 499 (1978).

[13] G.F. Leal Ferreira, Rev. Bras. Ens. Física 23, 252 (2001).

[14] J. Vaz Jr., Rev. Bras. Ens. Física 19, 234 (1997).

[15] B. Jancewicz, Multivectors and Clifford Algebra in Electrodynamics (World Scientific,1988). 